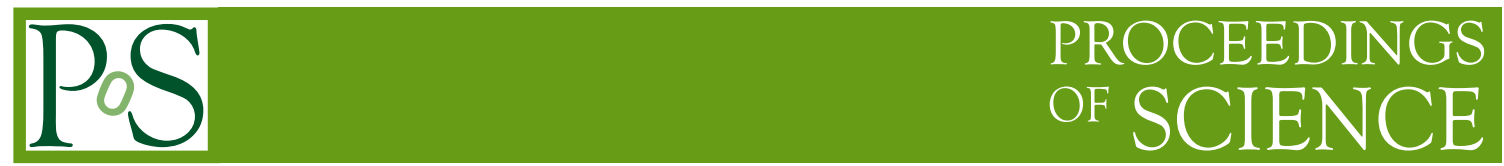

\title{
ATLAS measurements of CP violation with beauty mesons
}

\section{Radek Novotny ${ }^{a}$, on behalf of the ATLAS Collaboration}

${ }^{a}$ Department of Physics and Astronomy, University of New Mexico, Albuquerque, USA

E-mail: radek.novotny@cern.ch

The ATLAS experiment has performed measurements of the $C P$-violation phase $\phi_{s}$ and other physics parameters describing $B_{s}$ mixing in the $B_{s}^{0} \rightarrow J / \psi\left(\mu^{+} \mu^{-}\right) \phi\left(K^{+} K^{-}\right)$channel using data collected by the ATLAS detector from $13 \mathrm{TeV} p p$ collisions at the LHC with integrated luminosity of $80.5 \mathrm{fb}^{-1}$. The values measured from $13 \mathrm{TeV} p p$ collisions are then combined with those from $19.2 \mathrm{fb}^{-1}$ of $7 \mathrm{TeV}$ and $8 \mathrm{TeV}$ data. All measurements are in agreement with the Standard Model predictions as well as with other LHC measurements.

*** The European Physical Society Conference on High Energy Physics (EPS-HEP2021), ***

*** 26-30 July $2021 * * *$

*** Online conference, jointly organized by Universität Hamburg and the research center DESY ***

${ }^{*}$ Speaker 


\section{Introduction}

The measurement of the $C P$-violation phase, $\phi_{s}$, is performed in the $B_{s}^{0} \rightarrow J / \psi \phi$ channel where the $C P$-violation occurs due to interference between a direct decay and a decay with $B_{s}^{0}-\bar{B}_{s}^{0}$ mixing. The $B_{s}^{0} \rightarrow J / \psi \phi$ channel is used for this measurement due to its potential sensitivity to New Physics (NP). The NP processes could introduce additional contributions to the box diagrams describing the $B_{s}^{0}$ mixing and change the predicted $\phi_{s}$ value. There exist two predictions of the value of $\phi_{s}$ based on a combination of beauty and kaon physics observables, assuming no NP contributions to $B_{s}^{0}$ mixing and decays. The first prediction is done by the CKMFitter group with the value $\phi_{s}=-0.03696_{-0.00082}^{+0.00072} \mathrm{rad}$ [1], and according to the UTfit Collaboration the predicted value is $\phi_{s}=-0.03700 \pm 0.00104 \mathrm{rad}$ [2]. Although large NP enhancements of the mixing amplitude have been excluded by the precise measurement of the oscillation frequency, there is still some room on the order of the statistical uncertainty.

Other measured quantities in $B_{s}^{0}$ mixing are $\Delta \Gamma_{s}=\Gamma_{s}^{L}-\Gamma_{s}^{H}$ and $\Gamma_{s}=\left(\Gamma_{s}^{L}+\Gamma_{s}^{H}\right) / 2$, where $\Gamma_{s}^{L}$ and $\Gamma_{s}^{H}$ are the decay widths of the different mass eigenstates. Although, $\Delta \Gamma_{s}$ is not sensitive to $\mathrm{NP}$, the measurement is interesting for testing the SM prediction $\Delta \Gamma_{s}=0.091 \pm 0.013 \mathrm{ps}^{-1}$ [3].

This measurement uses the dataset of $80.5 \mathrm{fb}^{-1}$ of proton proton $(p p)$ collisions recorded by the ATLAS [4] detector at the LHC [5] during the period 2015 to 2017 at a centre-of-mass energy $\sqrt{s}=13 \mathrm{TeV}$. The statistical combination of measured parameters with the values extracted from the ATLAS Run1 measurements using $19.2 \mathrm{fb}^{-1}$ of $7 \mathrm{TeV}$ and $8 \mathrm{TeV}$ data was performed [6].

\section{The ATLAS detector}

The ATLAS detector [4] is a multi-purpose detector with a forward-backward symmetric cylindrical geometry and nearly $4 \pi$ coverage in solid angle, designed for exploration of NP in $p p$ collisions.

ATLAS consists of subdetectors grouped into three subsystems. The first group of detectors, called the inner tracking detector (ID), consists of a silicon pixel detector, a silicon microstrip detector, and a transition radiation tracker. The inner detector is designed to provide excellent momentum resolution for charged particles and both primary and secondary vertex position measurements with high precision in the pseudorapidity range $|\eta|<2.5$. It also provides electron identification over the region of $|\eta|<2.0$. An Insertable B-Layer [7] with a radius of $33 \mathrm{~mm}$ was installed between a new smaller beryllium beam pipe and the innermost pixel layer during Long Shutdown 1. The ID is surrounded by a thin superconducting solenoid providing a $2 \mathrm{~T}$ axial magnetic field, and by the second group of detectors. The high-granularity liquid-argon (LAr) sampling electromagnetic calorimeter and a steel/scintillator tile calorimeter provide coverage in the central rapidity range. The end-cap and forward regions are instrumented with LAr calorimeters for electromagnetic and hadronic measurements. The last group of detectors is the muon spectrometer (MS) that surrounds the calorimeters and consists of three large superconducting toroids with eight coils each, a system of muon tracking chambers, and fast detectors for triggering on muon tracks. 


\section{Reconstruction and candidate selection}

The events are collected with a mixture of triggers based on $J / \psi \rightarrow \mu^{+} \mu^{-}$identification, with muon $p_{\mathrm{T}}$ thresholds of either $4 \mathrm{GeV}$ or $6 \mathrm{GeV}$. Besides, each event must contain at least one reconstructed primary vertex, formed from at least four ID tracks, and at least one pair of oppositely charged muon candidates that are reconstructed using information from the MS and the ID. The pair of oppositely charged muon tracks is refitted to the common primary vertex and are accepted if $\chi^{2} /$ ndf $<10$. The candidates for the decay $\phi \rightarrow K^{+} K^{-}$are reconstructed from all pairs of oppositely charged tracks, with $p_{\mathrm{T}}>1 \mathrm{GeV}$ and $|\eta|<2.5$, that are not identified as muons. Candidate events for $B_{s}^{0}$ decays are selected by fitting the tracks for each combination of $J / \psi$ and $\phi$ to a common vertex and is accepted if $\chi^{2} / \mathrm{ndf}<3$ and $1.0085 \mathrm{GeV}<m\left(K^{+} K^{-}\right)<1.0305 \mathrm{GeV}$. The $B_{s}^{0}$ candidate with the lowest $\chi^{2} /$ ndf is selected in cases where more than one candidate passes all selections. In total, $2977526 B_{s}^{0}$ candidates is collected within the mass range of $5150-5650 \mathrm{MeV}$. For each $B_{s}^{0}$ meson candidate the proper decay time $t$ is estimated using:

$$
t=\frac{L_{x y} m_{B}}{p_{\mathrm{T}_{B}}}
$$

where $p_{\mathrm{T}_{B}}$ is the reconstructed transverse momentum of the $B_{s}^{0}$ meson candidate and $m_{B}$ denotes the mass of the $B_{s}^{0}$ meson, taken from Ref. [8]. The transverse decay length, $L_{x y}$, is the displacement in the transverse plane of the $B_{s}^{0}$ meson decay vertex with respect to the primary vertex, projected onto the direction of the $B_{s}^{0}$ transverse momentum. The primary vertex position is recalculated after removing any tracks used in the $B_{s}^{0}$ meson candidate to avoid biasing $L_{x y}$.

\section{Angular analysis and maximum likelihood fit}

This analysis is based on the full time-dependent angular analysis that is able to statistically separate $C P$-odd $(\mathrm{L}=1)$ and $C P$-even $(\mathrm{L}=0,2)$ amplitudes, which are the result of the pseudo-scalar to vector vector decay of $B_{s}^{0} \rightarrow J / \psi \phi$. The transversity basis is used for the description of the angles between final state particles.

The decay amplitudes are decomposed using three independent linear polarization states, $A_{0}$, $A_{\perp}$ and $A_{\|}$, of the vector mesons and are normalized such that $\left|A_{0}(0)\right|^{2}+\left|A_{\perp}(0)\right|^{2}+\left|A_{\|}(0)\right|^{2}=1$. For each of the transversity amplitudes there is an associated phase, $\delta_{0}=\arg \left(A_{0}\right), \delta_{\perp}=\arg \left(A_{\perp}\right)$ and $\delta_{\|}=\arg \left(A_{\|}\right)$. However, the primary signal is diluted by other processes with the same final state, such as non-resonant $B_{s} \rightarrow J / \psi K^{+} K^{-}$. These $\mathrm{S}$-wave states have to be counted in the final description of the decay due to their interference with the resonant $B_{S}^{0} \rightarrow J / \psi \phi$. The amplitude $A_{S}$ and phase $\delta_{S}$ are used to describe these S-wave states.

In order to extract physical parameters, an unbinned maximum likelihood fit to the $B_{s}^{0}$ candidates is performed. The fit uses information about the reconstructed mass $m$, the measured proper decay time $t$, the measured mass uncertainty $\sigma_{m}$, the measured proper decay time uncertainty $\sigma_{t}$, the tagging probability $P\left(B \mid Q_{x}\right)$, and the transversity angles $\Omega=\left(\theta_{\mathrm{T}}, \psi_{\mathrm{T}}, \phi_{\mathrm{T}}\right)$ of each $B_{s}^{0} \rightarrow J / \psi \phi$ decay candidate. The likelihood is defined as a combination of the signal Probability Density Function (PDF), combinatorial background PDF, distributions describing backgrounds from the channels $B_{d}^{0} \rightarrow J / \psi K^{* 0}, B_{d}^{0} \rightarrow J / \psi K \pi$ and $\Lambda_{b}^{0} \rightarrow J / \psi K p$ misidentified as $B_{s}^{0}$ candidates and 
a weighting factor to account for the trigger efficiency. A detailed description of the likelihood function and the systematic uncertainties can be found in Ref. [6].

\section{Flavour tagging}

To identify the flavor of the neutral $B_{s}^{0}$ meson at the point of production, the information from the opposite side of the $B_{s}^{0}$ decay is used. The opposite-side $b$-hadron is produced from $b \bar{b}$ pair production in $p p$ collisions. This method is called opposite-side tagging (OST).

In the present analysis, four types of taggers are used: two types of muons, electrons, and $b$-jets. All methods are based on the same discriminating variable, called the cone charge, which is defined as

$$
Q_{x}=\frac{\sum_{i}^{N \text { tracks }} q_{i} \cdot\left(p_{\mathrm{T} i}\right)^{\kappa}}{\sum_{i}^{N \text { tracks }}\left(p_{\mathrm{T} i}\right)^{\kappa}},
$$

where $x=\{\mu, e$, jet $\}$ refers to muon, electron, or jet charge. The summation is made over a selected set of tracks in a cone, $\Delta R=\sqrt{(\Delta \phi)^{2}+(\Delta \eta)^{2}}<0.5$, around the lepton or jet direction. The constant $\kappa$ is found empirically for each tagging method, respectively.

The calibration of each tagging method uses data containing $B^{ \pm} \rightarrow J / \psi K^{ \pm}$candidate decays. The charge of the kaon in a $B^{ \pm} \rightarrow J / \psi K^{ \pm}$decay determines the initial flavour of the $b$-meson and gives us the cone charge probability distribution $P\left(Q_{x} \mid B^{ \pm}\right)$. This probability is then used for the calculation of the probability to tag a $B_{s}^{0}$ meson containing a $\bar{b}$-quark as $P\left(B \mid Q_{x}\right)=P\left(Q_{x} \mid B^{+}\right) /\left(P\left(Q_{x} \mid B^{+}\right)+P\left(Q_{x} \mid B^{-}\right)\right)$.

The performance of each tagging method is represented by a tagging power defined as $T_{x}=\sum_{i} \epsilon_{x i} \cdot \mathcal{D}^{2}\left(Q_{x i}\right)$, where the $\epsilon_{x}$ is the tagging efficiency, $\mathcal{D}\left(Q_{x}\right)$ is the dilution, and the sum is over the probability distribution in intervals of the cone charge variable.

The efficiency, $\epsilon_{x}$, of an individual tagging method is defined as the fraction of signal events tagged by that method compared to the total number of signal events in the sample. The dilution is defined as $\mathcal{D}\left(Q_{x}\right)=2 P\left(B \mid Q_{x}\right)-1$ and represents the purity of a particular flavour tagging method. An effective dilution, $D_{x}=\sqrt{T_{x} / \epsilon_{x}}$, is calculated from the measured tagging power and efficiency. The summary of the tagging performance for each tagging method is given in Table1.

Table 1: Summary of tagging performances on the sample of $B^{ \pm} \rightarrow J / \psi K^{ \pm}$signal candidates for each tagging method. Taken from [6].

\begin{tabular}{l|c|c|c}
\hline Tag method & $\epsilon_{x}[\%]$ & $D_{x}[\%]$ & $T_{x}[\%]$ \\
\hline \hline Tight muon & $4.50 \pm 0.01$ & $43.8 \pm 0.2$ & $0.862 \pm 0.009$ \\
Electron & $1.57 \pm 0.01$ & $41.8 \pm 0.2$ & $0.274 \pm 0.004$ \\
Low- $p_{\mathrm{T}}$ muon & $3.12 \pm 0.01$ & $29.9 \pm 0.2$ & $0.278 \pm 0.006$ \\
Jet & $12.04 \pm 0.02$ & $16.6 \pm 0.1$ & $0.334 \pm 0.006$ \\
\hline Total & $21.23 \pm 0.03$ & $28.7 \pm 0.1$ & $1.75 \pm 0.01$ \\
\hline
\end{tabular}

\section{Results}

The results of the likelihood fit for the parameters of interest are shown in Table 2. While for most of the physics parameters, including $\phi_{s}, \Delta \Gamma_{s}$ and $\Gamma_{s}$, the fit determines a single solution 
with Gaussian behavior of the projection of the log-likelihood, for the strong-phases $\delta_{\perp}$ and $\delta_{\|}$ two well-separated local maxima of the likelihood are found. The difference in $-2 \Delta \ln (L)$ between the two solutions is 0.03 , favouring (a) but without ruling out (b). The two-fold behavior of the likelihood in the strong phases is the result of an approximate symmetry of the signal PDF, where the signal PDF is invariant under the transformation

$$
\left\{\delta_{\|}, \delta_{\perp}, \delta_{S}\right\} \rightarrow\left\{2 \pi-\delta_{\|}, \delta_{\perp}+2\left(\pi-\delta_{\|}\right), \delta_{S}+2\left(\pi-\delta_{\|}\right)\right\}
$$

Table 2: Fitted values for the physical parameters of interest with their statistical and systematic uncertainties. For variables $\delta_{\perp}$ and $\delta_{\|}$the values are given for the two solutions (a) and (b). The difference in $-2 \Delta \ln (L)$ between solutions (b) and (a) is 0.03 . For the rest of the variables, the values for the two minima are consistent. The same is true for the statistical and systematic uncertainties of all the variables. Taken from [6].

\begin{tabular}{c|c|c|c}
\hline Parameter & Value & $\begin{array}{c}\text { Statistical } \\
\text { uncertainty }\end{array}$ & $\begin{array}{c}\text { Systematic } \\
\text { uncertainty }\end{array}$ \\
\hline \hline$\phi_{s}[\mathrm{rad}]$ & -0.081 & 0.041 & 0.022 \\
$\Delta \Gamma_{s}\left[\mathrm{ps}^{-1}\right]$ & 0.0607 & 0.0047 & 0.0043 \\
$\Gamma_{S}\left[\mathrm{ps}^{-1}\right]$ & 0.6687 & 0.0015 & 0.0022 \\
$\left|A_{\|}(0)\right|^{2}$ & 0.2213 & 0.0019 & 0.0023 \\
$\left|A_{0}(0)\right|^{2}$ & 0.5131 & 0.0013 & 0.0038 \\
$\left|A_{S}(0)\right|^{2}$ & 0.0321 & 0.0033 & 0.0046 \\
$\delta_{\perp}-\delta_{S}[\mathrm{rad}]$ & -0.25 & 0.05 & 0.04 \\
\hline \multicolumn{3}{|c}{ Solution (a) } \\
$\delta_{\perp}[\mathrm{rad}]$ & 3.12 & 0.11 & 0.06 \\
$\delta_{\|}[\mathrm{rad}]$ & 3.35 & 0.05 & 0.09 \\
\hline \multicolumn{3}{|c}{ Solution (b) } \\
$\delta_{\perp}[\mathrm{rad}]$ & 2.91 & 0.11 & 0.06 \\
$\delta_{\|}[\mathrm{rad}]$ & 2.94 & 0.05 & 0.09 \\
\hline
\end{tabular}

The parameters obtained are combined with results from ATLAS Run1 measurements using a Best Linear Unbiased Estimator method (BLUE). This method uses the measured values and uncertainties of the parameters as well as the correlations between them. The combined values of parameters are shown in Table 3. The two-dimensional contours in the $\phi_{s}-\Delta \Gamma_{s}$ plane for the combined measurement in comparison with other experiments are shown in Figure 1. 
Table 3: Combined values for the physical parameters of interest with the statistical and systematic uncertainties for both solutions. Taken from [6].

\begin{tabular}{c||c|c|c||c|c|c}
\hline \multicolumn{1}{c||}{ Parameter } & \multirow{3}{*}{ Value } & $\begin{array}{c}\text { Solution (a) } \\
\text { Statistical } \\
\text { uncertainty }\end{array}$ & $\begin{array}{c}\text { Systematic } \\
\text { uncertainty }\end{array}$ & Value & $\begin{array}{c}\text { Solution (b) } \\
\text { Stistical } \\
\text { uncertainty }\end{array}$ & $\begin{array}{c}\text { Systematic } \\
\text { uncertainty }\end{array}$ \\
\hline \hline$\phi_{S}[\mathrm{rad}]$ & -0.087 & 0.036 & 0.021 & -0.087 & 0.036 & 0.021 \\
$\Delta \Gamma_{S}\left[\mathrm{ps}^{-1}\right]$ & 0.0657 & 0.0043 & 0.0037 & 0.0657 & 0.0043 & 0.0037 \\
$\Gamma_{S}\left[\mathrm{ps}^{-1}\right]$ & 0.6703 & 0.0014 & 0.0018 & 0.6704 & 0.0014 & 0.0018 \\
$\left|A_{\|}(0)\right|^{2}$ & 0.2220 & 0.0017 & 0.0021 & 0.2218 & 0.0017 & 0.0021 \\
$\left|A_{0}(0)\right|^{2}$ & 0.5152 & 0.0012 & 0.0034 & 0.5152 & 0.0012 & 0.0034 \\
$\left|A_{S}\right|^{2}$ & 0.0343 & 0.0031 & 0.0045 & 0.0348 & 0.0031 & 0.0045 \\
$\delta_{\perp}[\mathrm{rad}]$ & 3.22 & 0.10 & 0.05 & 3.03 & 0.10 & 0.05 \\
$\delta_{\|}[\mathrm{rad}]$ & 3.36 & 0.05 & 0.09 & 2.95 & 0.05 & 0.09 \\
$\delta_{\perp}-\delta_{S}[\mathrm{rad}]$ & -0.24 & 0.05 & 0.04 & -0.24 & 0.05 & 0.04 \\
\hline
\end{tabular}

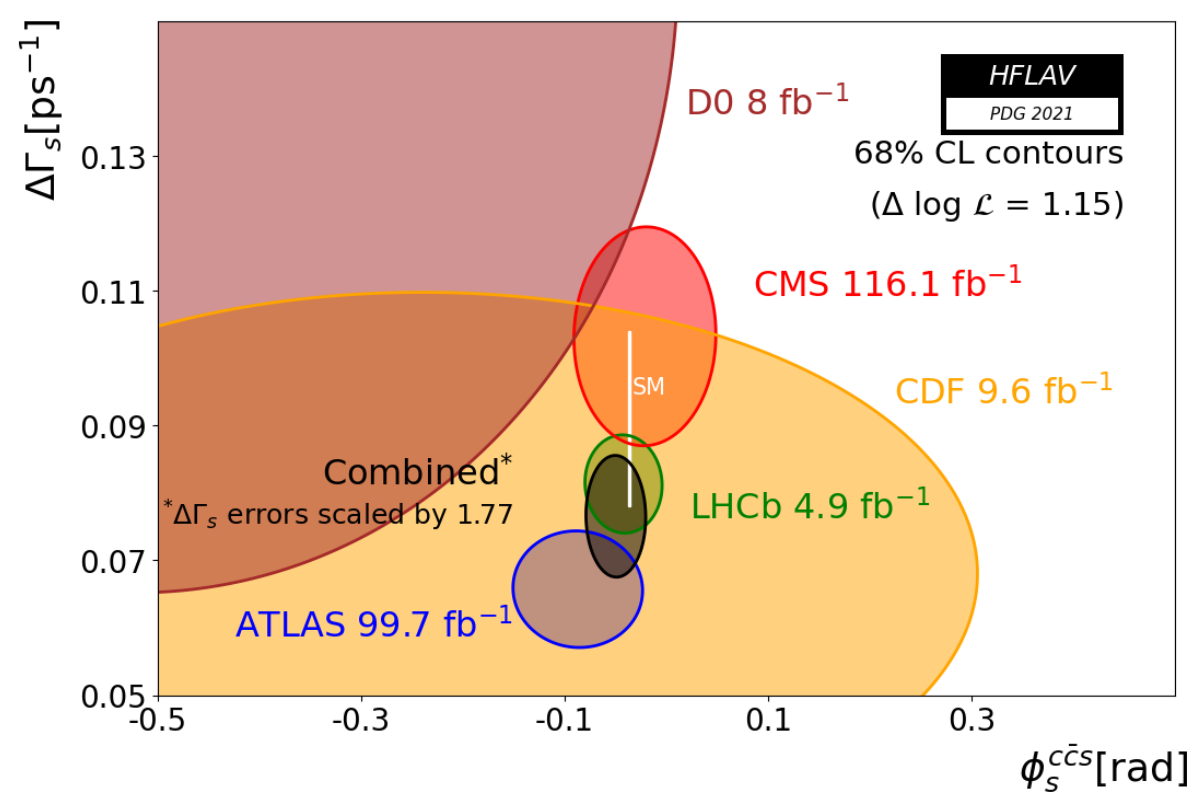

Figure 1: The $68 \%$ confidence level likelihood contours in the $\phi_{s}-\Delta \Gamma_{s}$ plane, showing measurements by ATLAS, CMS, CDF, D0 and LHCb, and their combined contour (black solid line and shaded area), as well as the Standard Model predictions (very thin white rectangle). Taken from [9].

\section{Summary}

A measurement of the time-dependent $C P$ violation parameters in $B_{s}^{0} \rightarrow J / \psi\left(\mu^{+} \mu^{-}\right) \phi\left(K^{+} K^{-}\right)$ decays from a data sample of $p p$ collisions at a centre-of-mass energy $\sqrt{s}=13 \mathrm{TeV}$ collected with the ATLAS detector between years 2015 and 2017 with integrated luminosity of $80.5 \mathrm{fb}^{-1}$ is presented. The values from the $13 \mathrm{TeV}$ analysis are consistent with those obtained in the previous analysis using $7 \mathrm{TeV}$ and $8 \mathrm{TeV}$ ATLAS data. The two measurements are statistically combined, 
leading to the following results:

$$
\begin{aligned}
\phi_{s} & =-0.087 \pm 0.036 \text { (stat.) } \pm 0.021 \text { (syst.) } \mathrm{rad} \\
\Delta \Gamma_{s} & =0.0657 \pm 0.0043 \text { (stat.) } \pm 0.0037 \text { (syst.) } \mathrm{ps}^{-1} \\
\Gamma_{s} & =0.6703 \pm 0.0014 \text { (stat.) } \pm 0.0018 \text { (syst.) } \mathrm{ps}^{-1}
\end{aligned}
$$

The new results from the ATLAS measurement on the $C P$ violation phase $\phi_{s}$ in the $B_{s}^{0} \rightarrow J / \psi \phi$ channel are consistent with Standard Model predictions as well as with other LHC measurements.

\section{References}

[1] J. Charles et al., Current status of the Standard Model CKM fit and constraints on $\Delta F=2$ New Physics, Phys. Rev. D 91 (2015) 073007 [1501.05013].

[2] UTFIT collaboration, The Unitarity Triangle Fit in the Standard Model and Hadronic Parameters from Lattice QCD: A Reappraisal after the Measurements of $\Delta m(s)$ and $B R(B \rightarrow \tau v(\tau))$, JHEP 10 (2006) 081 [hep-ph/0606167].

[3] A. Lenz and U. Nierste, Numerical Updates of Lifetimes and Mixing Parameters of B Mesons, in 6th International Workshop on the CKM Unitarity Triangle, 2, 2011 [1102 . 4274].

[4] ATLAS collaboration, The ATLAS Experiment at the CERN Large Hadron Collider, JINST 3 (2008) S08003.

[5] L. Evans and P. Bryant, eds., LHC Machine, JINST 3 (2008) S08001.

[6] ATLAS collaboration, Measurement of the CP-violating phase $\phi_{s}$ in $B_{s}^{0} \rightarrow J / \psi \phi$ decays in ATLAS at 13 TeV, Eur. Phys. J. C 81 (2021) 342 [2001. 07115].

[7] ATLAS IBL collaboration, Production and Integration of the ATLAS Insertable B-Layer, JINST 13 (2018) T05008 [1803.00844].

[8] Particle Data Group collaboration, Review of Particle Physics, Phys. Rev. D 98 (2018) 030001.

[9] HFLAV collaboration, Averages of b-hadron, c-hadron, and $\tau$-lepton properties as of 2018, Eur. Phys. J. C 81 (2021) 226 [1909.12524]. 